\title{
Inborn Errors of Metabolism: The Achilles' Heel of the Respiratory System
}

\author{
Dominique Valeyre ${ }^{a, b}$ Nadia Nathan ${ }^{c, d}$ Jean-François Bernaudin ${ }^{b, d}$ \\ a Université Paris 13, Sorbonne-Paris-Cité, EA2363, and b AP-HP, Département de Pneumologie, Hôpital Avicenne, \\ Bobigny, and ${ }^{\mathrm{C}}$ AP-HP Service de Pneumologie Pédiatrique, Hôpital Armand-Trousseau, and d Sorbonne Universités, \\ Université Pierre et Marie Curie, Paris, France
}

Achilles, the great hero of the Trojan War, was the son of the sea nymph Thetis and of Peleus, the human king of the Myrmidons. To compensate for his human vulnerability, Thetis conceived for Achilles an invincible protection by dipping him in the Styx river. However, as she held him by the heel, this part of his body did not get any shield and, thus, 2 decades later when Paris shot him with an arrow in the unprotected heel...

There is no question that cystic fibrosis represents the oldest known and most frequent respiratory disease due to inborn aberrations, which is in most cases revealed by an early onset but could also be discovered in adult life [1]. Recently, more attention has been paid to surfactant protein dysfunctions, a significant cause of interstitial lung diseases in children [2]. Such aberrations are now recognized as predisposing factors for adult lung interstitial fibrosis and even for lung cancer [2], while several other diseases, among them telomere-related lung fibrosis or genetic pulmonary arterial hypertension, are prone to be discovered in adulthood $[3,4]$.

The review by Tran et al. [5] has the important merit of drawing attention to under-recognized entities in adults, those due to inborn errors of metabolism. Interestingly, we are reminded that the lung functions are not limited to respiration but also include major metabolic

\section{KARGER}

(C) 2017 S. Karger AG, Basel

E-Mail karger@karger.com

www.karger.com/res roles. As the respiratory presentation of inborn errors of metabolism may be the same as that of far more common alternative diseases, clinicians may overlook these diseases for a long time. Useless treatment may be applied, although for some diseases, innovative treatments could help patients and prevent irreversible damage. Furthermore, for some of these diseases, innovative treatment trials based on pathophysiology are still needed in the near future.

There is already substantial literature focused on the respective diseases covered by the review, from NiemannPick diseases to mitochondrial diseases, but this literature is dedicated to pediatricians and not to physicians treating adults. Therefore, the original approach of this review is to consider these rare diseases from a point of view of physicians who treat adults and to share that knowledge with clinicians insufficiently aware of these diseases. Moreover, it is particularly noteworthy that this complete review presents all the diseases associated with inborn errors of metabolism and classifies them according to a pathophysiological perspective. The reported disorders, namely those involving complex molecules (sphingolipidoses, glycogen storage diseases, and mucopolysaccharidosis), disorders of small molecules that give rise to intoxication, or disorders involving energy metabolism, are

Dominique Valeyre

Hôpital Avicenne

125 Rue de Stalingrad

FR-93009 Bobigny (France)

E-Mail dominique.valeyre@aphp.fr 
presented from their pathophysiology to clinical presentation, diagnosis criteria, and treatment.

Pulmonary-induced diseases due to inborn errors of metabolism show very diverse presentations: infiltrative lung diseases, airflow obstruction, obstructive sleep apnea syndrome, pulmonary hypertension, or respiratory myopathies. Often, adults suffering from these diseases have had their diagnosis established in childhood, and physicians treating adults should know how to take care of patients with these diseases. In some patients, particularly those with a late-onset lung disease, the diagnosis is made when they are adults, and the question is how their physicians could come up with such a diagnosis? The presence of hepatosplenomegaly and neurologic manifestations associated with unexplained infiltrative lung disease should suggest an inborn error of metabolism. The same applies to unexplained interstitial lung diseases with crazy paving, unclear obstructive sleep apnea syndrome, respiratory myopathy, pulmonary hypertension, and hyperventilation. Physicians treating adults should learn how to confirm the diagnosis using blood biochemistry, genetic investigations, and sometimes pathology. For some diseases, treatments based on enzyme replacement therapy, for instance in type I Gaucher disease, are effective, while, for others, enrolment in pathophysiological-based treatment trials may be a good opportunity.
For example, sphingolipidoses are among the more common inborn errors of metabolism behind lung diseases observed in adults. Niemann-Pick disease type B is remarkable due to the conjunction of splenomegaly and dyspnea. Almost all adults with Niemann-Pick type B suffer from infiltrative lung disease at some point, and this pulmonary involvement is often at the forefront, with major morbidity and mortality. Enzyme replacement therapy is a promising treatment, while acid sphingomyelinase is under trial. Moreover, it should be recollected that upper airway disease is common in mucopolysaccharidoses.

Multidisciplinary discussions involving pulmonologists, radiologists, and pathologists are currently the standard for diagnosis and treatment of difficult interstitial lung diseases [6]. For adults with pulmonary involvement induced by inborn errors of metabolism, adequate multidisciplinary teams might include, in addition to pulmonologists, metabolic physicians and pediatricians specialized in this medical field.

In conclusion, we agree with Tran et al. [5] that "clinical suspicion, early recognition, prompt diagnosis and appropriate care of respiratory manifestations are crucial," and therefore, their review is a particularly important one.

\section{References}

Inborn Errors of Metabolism
1 Elborn JS: Cystic fibrosis. Lancet 2016;388: 2519-2531.

-2 Van Moorsel CH, Hoffman TW, van Batenburg AA, Klay D, van der Vis JJ, Grutters JC: Understanding idiopathic interstitial pneumonia: a gene-based review of stressed lungs. Biomed Res Int 2015;2015:304186.

-3 Newton CA, Batra K, Torrealba J, et al: Telomere-related lung fibrosis is diagnostically heterogeneous but uniformly progressive. Eur Respir J 2016;48:1710-1720.

4 Austin ED, Loyd JE: The genetics of pulmonary arterial hypertension. Circ Res 2014;115: 189-202.
5 Tran C, Barbey F, Lazor R, Bonafé L: Pulmonary involvement in adult patients with inborn errors of metabolism. Respiration 2017;94:2-13.

-6 Travis WD, Costablel U, Hansell DM, et al: An official American Thoracic Society/European Respiratory Society statement: update of the international multidisciplinary classification of the idiopathic interstitial pneumonias. Am J Respir Crit Care Med 2013;188:733748. 